\title{
The Permanental Polynomials of Subdivision Graphs
}

\author{
Wei Li
}

\begin{abstract}
Graph polynomials are important objects of research in graph theory. Particularly, the permanental polynomials are widely used in Physics and Chemistry. As the difficulty to evaluate the permanental polynomials, this paper deals with the computation of the permanental polynomials of graphs under various operations. Firstly, we give explicit expressions for the permanental polynomials of single subdivision graphs and bisubdivision graphs in recursive ways, respectively. Then we deduce the permanental polynomials of degree subdivision graphs by the product of matrices. Based on these, the permanental polynomials of those physical graphs and chemical graphs which can be generated by subdivision operations can be derived.
\end{abstract}

Index Terms-Permanent, permanental polynomial, subdivision graph.

\section{INTRODUCTION}

The permanental polynomials of graphs originate from Mathematics. Recently, they have attracted some interest in Chemistry, Physics and graph theory. For example, the Jones polynomial, which has deep connections with statistical mechanics, can be expressed as the permanent of a matrix [1]. Moreover, the computation of the transition amplitude of a quantum circuit can also be encoded as computing the permanent of a matrix [2]. In addition, the constant term of the permanental polynomial of a chemical graph enumerates the close-packed dimers (which is termed as perfect matchings in Mathematics) of a graph, and the coefficients and zeros of permanental polynomials are related to the stability and structure information of chemical graphs [3], [4]. Therefore, it is interesting and exciting to evaluate the permanental polynomials of graphs.

As is well known, computing the permanent of a matrix is a \#P-complete problem [5]. So it is very hard to compute the permanents and the permanental polynomials directly. Is there an efficient method to deal with the permanental polynomials of some interesting and special graphs? Many graphs widely used in Chemistry and Physics could be generated by a series of subdivision operations. Motivated by this, in this paper we provide ways to compute the permanental polynomials of subdivision graphs. We introduce some definitions and notations.

A graph $G$ is a triple consisting of a vertex set $V$, an edge set $E$, and a relation that associates with each edge two vertices called the end-vertices. An edge $e$ with end-vertices

Manuscript received May 12, 2014; revised July 12, 2014. This work is supported by the Northwestern Polytechnical University Foundation for Fundamental Research and the Scientific Research Foundation of Northwestern Polytechnical University (grant no. GDKY1002, 13GH0313).

Wei Li is with the Department of Applied Mathematics, Northwestern Polytechnical University, Xi'an 710129, P. R. China (e-mail: liw@nwpu.edu.cn). $u$ and $v$ is denoted by $e=(u, v)$. A cycle is a graph with an equal number of vertices and edges whose vertices can be placed around a circle so that two vertices are joined by an edge if and only if they appear consecutively along the circle.

Let $G$ be a finite and simple graph on $n$ vertices. The permanental polynomial of $G$ is defined as

$$
\pi(G, x)=\operatorname{per}(x I-A(G))=\sum_{k=0}^{n} b_{k} x^{n-k}
$$

where $I$ is the identity matrix of order $n, A(G)$ is the adjacency matrix of $G$ and the permanent $\operatorname{per}(A)$ of a matrix $A=\left(a_{i j}\right)_{n \times n}$ is given as [6]

$$
\operatorname{per}(A)=\sum_{\sigma \in \Lambda_{n}} \prod_{i=1}^{n} a_{i \sigma(i)}
$$

with $\Lambda_{n}$ denoting the set of all the permutations of $\{1,2, \ldots, n\}$.

In the literatures [7], [8], they proved that the coefficient of the permanental polynomial satisfies that

$$
(-1)^{i} b_{i}=\sum_{H} 2^{\kappa(H)}
$$

where the sum ranges over all subgraphs $H$ on $i$ vertices whose components are single edges or cycles, and $\kappa(H)$ is the number of cycles. Based on this result, Borowiecki and Jóźwiak [9] studied the relationship between the permanental polynomial of a graph and the permanental polynomials of its subgraphs, and they obtained the following results.

Theorem I.1 [9] Let $e=(u, v)$ be an edge of a graph $G$ and $\Gamma_{e}(G)$ the set of cycles containing $e$. Then

$$
\begin{gathered}
\pi(G, x)=\pi(G-e, x)+\pi(G-u-v, x) \\
+2 \sum_{C \in \Gamma_{e}(G)}(-1)^{|V(C)|} \pi(G-V(C), x),
\end{gathered}
$$

where $C$ is a cycle in $\Gamma_{e}(G)$ and $|V(C)|$ denotes the number of vertices of $C$.

Theorem I.2 [9] Let $u$ be a vertex of a graph $G$ and $\Gamma_{u}(G)$ the set of cycles containing $u$. Then

$$
\begin{gathered}
\pi(G, x)=x \pi(G-u, x)+\sum_{v \sim u} \pi(G-u-v, x) \\
+2 \sum_{C \in \Gamma_{u}(G)}(-1)^{|V(C)|} \pi(G-V(C), x),
\end{gathered}
$$


where $v \sim u$ means $v$ and $u$ are the end-verices of an edge, $C$ is a cycle in $\Gamma_{u}(G)$ and $|V(C)|$ is the number of vertices of $C$.

Theorems I.1 and I.2 provide ways to deduce the permanental polynomials, but it is not convenient to use. On the purpose to obtain the permanental polynomials easily and efficiently, we turn to derive the permanental polynomials of graphs in a linear algebra method. Explicitly, we will deduce the permanental polynomials of graphs in a recursive way by the product of matrices.

The organization of this paper is as follows. In Section II we give the expressions of the permanental polynomials of a single subdivision graph and a bisubdivision graph, respectively. In Section III we obtain the permanental polynomials of degree subdivision graphs by the product of matrices. These theoretical results provide methods to compute the permanental polynomial of graphs under subdivision operations.

\section{The Permanental Polynomials of GRAPHS By SUBDIVIDING AN EDGE OF A GRAPH}

\section{A. The Permanental Polynomials of Single Subdivision Graphs}

Let $H$ be the graph with $m$ edges $e_{1}, e_{2}, \ldots, e_{m}$. If a graph $G$ can be obtained from $H$ by breaking up each $e_{i}$ into $k_{i}+1$ segments by inserting $k_{i}$ intermediate vertices between its two end-vertices, then $G$ is said to be a subdivision graph of $H$. For a prescribed edge $e_{j}$ of graph $H$, if $k_{j}=1$, then $G$ is said to be a single subdivision of $H$ by $e_{j}$; if $k_{j}=2$, then $G$ is said to be a bisubdivision of $H$ by $e_{j}$, see Fig. 1 .

A subdivision graph of $H$ can be obtained from $H$ by a series of single subdivisions or bisubdivisions. In the following, we will deduce the permanental polynomials of the single subdivision of $H$ and the bisubdivision of $H$, respectively.

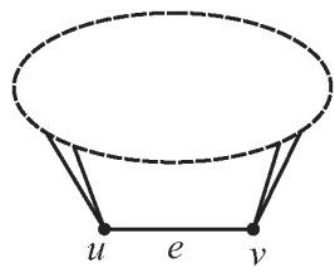

a)

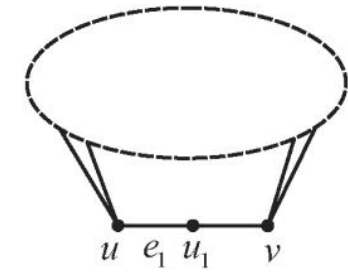

b)

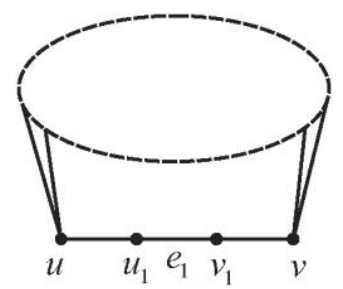

c)

Fig. 1. a) $H$, b) the single subdivision of $H$ and c) the bisubdivision of $H$.

Theorem II.1 Let $e=(u, v)$ be an edge of $H$ and $G$ the single subdivision of $H$ by inserting one intermediate vertex $u_{1}$ between $u$ and $v$. Denote the edge $\left(u, u_{1}\right)$ in $G$ by $e_{1}$ Let $\alpha(H, e, u, v)$ be the column vector $(\pi(H, x)$, $\pi(H-e, x), \pi(H-u, x), \pi(H-v, x), \pi(H-u-v, x))^{T}$.

Then

$$
\alpha\left(G, e_{1}, u, u_{1}\right)=A_{1} \cdot \alpha(H, e, u, v),
$$

where $A_{1}$ is a $5 \times 5$ matrix whose $i$-th row vector is $r_{i}$ for $1 \leq i \leq 5 . \quad$ Explicitly, $\quad r_{1}=(-1, x+1,1,1,1) \quad$; $r_{2}=(0, x, 0,1,0) ; r_{3}=(0,0, x, 0,1) ; r_{4}=(0,1,0,0,0)$; $r_{5}=(0,0,1,0,0)$.

Proof: According to Theorem I.2,

$$
\begin{aligned}
\pi(G, x)= & x \pi\left(G-u_{1}, x\right)+\pi\left(G-u_{1}-u, x\right)+\pi\left(G-u_{1}-v, x\right) \\
& +2 \sum_{C \in \Gamma_{u_{1}}(G)}(-1)^{|V(C)|} \pi(G-V(C), x) \\
= & x \pi(H-e, x)+\pi(H-u, x)+\pi(H-v, x) \\
& -2 \sum_{C \in \Gamma_{e}(H)}(-1)^{|V(C)|} \pi(H-V(C), x)
\end{aligned}
$$

Following Theorem I.1, it holds that

$$
\begin{gathered}
\pi(H, x)=\pi(H-e, x)+\pi(H-u-v, x) \\
+2 \sum_{C \in \Gamma_{e}(H)}(-1)^{|V(C)|} \pi(H-V(C), x) .
\end{gathered}
$$

Combining (1), we obtain that

$$
\begin{gathered}
\pi(G, x)=-\pi(H, x)+(x+1) \pi(H-e, x)+\pi(H-u, x) \\
+\pi(H-v, x)+\pi(H-u-v, x) .
\end{gathered}
$$

Applying Theorem I.1, we get that

$$
\begin{gathered}
\pi(G-u, x)=x \pi\left(G-u-u_{1}, x\right)+\pi\left(G-u-u_{1}-v, x\right) \\
=x \pi(H-u, x)+\pi(H-u-v, x)
\end{gathered}
$$

and

$$
\begin{aligned}
\pi\left(G-e_{1}, x\right) & =x \pi\left(G-e_{1}-u_{1}, x\right)+\pi\left(G-e_{1}-u_{1}-v, x\right) \\
& =x \pi(H-e, x)+\pi(H-v, x) .
\end{aligned}
$$

$$
\begin{array}{llr}
\text { It } \quad \text { is } \quad \text { easy to } & \text { see } & \text { that } \\
\pi\left(G-u_{1}, x\right)=\pi(H-e, x) & & \text { and } \\
\pi\left(G-u-u_{1}, x\right)=\pi(H-u, x) . & & \text { Thus } \\
\alpha\left(G, e_{1}, u, u_{1}\right)=A_{1} \cdot \alpha(H, e, u, v) \text { follows. } &
\end{array}
$$

\section{B. The Permanental Polynomials of Bisubdivision Graphs}

Theorem II.2 Let $e=(u, v)$ be an edge of $H$ and $G$ the bisubdivision of $H$ by inserting two intermediate vertices $u_{1}$ and $v_{1}$ between $u$ and $v$. Denote the edge $\left(u_{1}, v_{1}\right)$ in $G$ by $e_{1}$. Let $\alpha(H, e, u, v)$ be the column vector $(\pi(H, x)$, 
$\pi(H-e, x), \pi(H-u, x), \pi(H-v, x), \pi(H-u-v, x))^{T}$.

Then

$$
\alpha\left(G, e_{1}, u_{1}, v_{1}\right)=A_{2} \cdot \alpha(H, e, u, v)
$$

where $A_{2}$ is a $5 \times 5$ matrix whose $i$-th row vector is $c_{i}$ for $1 \leq i \leq 5$. Explicitly, $c_{1}=\left(1, x^{2}, x, x, 0\right)$; $c_{2}=\left(0, x^{2}, x, x, 1\right) \quad ; \quad c_{3}=(0, x, 0,1,0)$ $c_{4}=(0, x, 1,0,0) ; c_{5}=(0,1,0,0,0)$.

Proof: By Theorem I.1, it holds that

$$
\begin{gathered}
\pi(G, x)=\pi\left(G-e_{1}, x\right)+\pi\left(G-u_{1}-v_{1}, x\right) \\
+2 \sum_{C \in \Gamma_{e_{1}}(G)}(-1)^{|V(C)|} \pi(G-V(C), x) \\
=\pi\left(G-e_{1}, x\right)+\pi(H-e, x) \\
+2 \sum_{C \in \Gamma_{e}(H)}(-1)^{|V(C)|} \pi(H-V(C), x) .
\end{gathered}
$$

Similarly,

$$
\begin{gathered}
\pi\left(G-e_{1}, x\right) \\
=\pi\left(G-e_{1}-\left(u, u_{1}\right), x\right)+\pi\left(G-e_{1}-u-u_{1}, x\right) \\
=x \pi\left(G-u_{1}, x\right)+\pi\left(G-u_{1}-u, x\right) \\
=x\left[x \pi\left(G-u_{1}-v_{1}, x\right)+\pi\left(G-u_{1}-v_{1}-v, x\right)\right] \\
+x \pi\left(G-u_{1}-u-v_{1}, x\right)+\pi\left(G-u_{1}-u-v_{1}-v, x\right) \\
=x^{2} \pi(H-e, x)+x \pi(H-v, x)+x \pi(H-u, x) \\
+\pi(H-u-v, x) .
\end{gathered}
$$

In the same way, we can get that

$$
\begin{aligned}
& \pi\left(G-u_{1}, x\right)=x \pi(H-e, x)+\pi(H-v, x) \\
& \pi\left(G-v_{1}, x\right)=x \pi(H-e, x)+\pi(H-u, x) \\
& \pi\left(G-u_{1}-v_{1}, x\right)=\pi(H-e, x) .
\end{aligned}
$$

Substituting $\quad 2 \sum_{C \in \Gamma_{e}(H)}(-1)^{|V(C)|} \pi(H-V(C), x) \quad$ in

for $\pi(H, x)-\pi(H-e, x)-\pi(H-u-v, x)$ and combining (3), we obtain that

$$
\begin{aligned}
& \pi(G, x)=\pi(H, x)+x^{2} \pi(H-e, x) \\
& +x[\pi(H-u, x)+\pi(H-v, x)] .
\end{aligned}
$$

Thus we derive that $\alpha\left(G, e_{1}, u_{1}, v_{1}\right)=A_{2} \cdot \alpha(H, e, u, v)$.

Remark II.3 Based on Theorems II. 1 and II.2, if $G$ is a subdivision of $H$ by subdividing some edge $e$, then the permanental polynomials of $G$ and its subgraphs can be derived by the permanental polynomials of $H$ and its subgraphs in a recursive way.

\section{Examples}

Fig. 2 (a) is the complete bipartite graph $K_{2,3}$ and $e$ is the edge joining $u_{1}$ and $v_{1} . G_{1}$ is the graph obtained from $K_{2,3}$ by inserting two vertices $w_{1}$ and $w_{2}$ between $u_{1}$ and $v_{1}$ and the edge $\left(w_{1}, w_{2}\right)$ is denoted by $e_{1} . G_{2}$ is the graph obtained from $G_{1}$ by inserting one vertex $z_{1}$ between $w_{1}$ and $w_{2}$. Let $e_{2}=\left(w_{1}, z_{1}\right)$ (see Fig. 2 (b) and Fig. 2 (c) ). Since

$\alpha\left(K_{2,3}, e, u_{1}, v_{1}\right)=\left(x^{5}+6 x^{3}+12 x, x^{5}+5 x^{3}+6 x, x^{4}+3 x^{2}\right.$, $\left.x^{4}+4 x^{2}+4, x^{3}+2 x\right)$, the permanental polynomials of $G_{1}, G_{2}$ and their subgraphs can be derived as below.

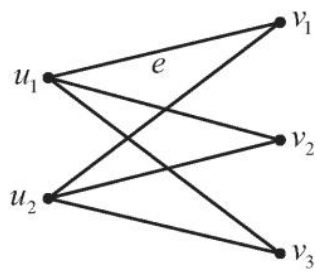

a)

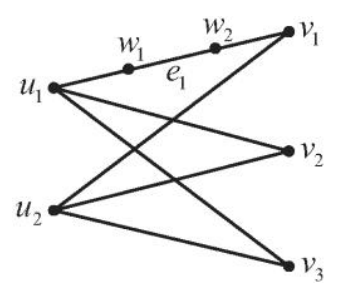

b)

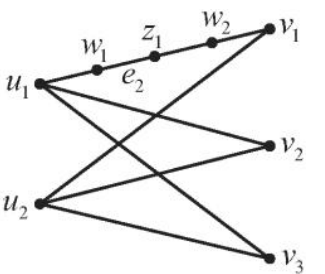

c)
Fig. 2. (a) $K_{2,3}$, (b) $G_{1}$, and (c) $G_{2}$.

TABLE I: THE PERMANENTAL POLYNOMIALS OF $G_{1}$ AND ITS SUBGRAPHS

\begin{tabular}{ll}
\hline$\pi\left(G_{1}, x\right)$ & $x^{7}+8 x^{5}+19 x^{3}+16 x$ \\
$\pi\left(G_{1}-e_{1}, x\right)$ & $x^{7}+7 x^{5}+14 x^{3}+6 x$ \\
$\pi\left(G_{1}-u_{1}, x\right)$ & $x^{6}+6 x^{4}+10 x^{2}+4$ \\
$\pi\left(G_{1}-v_{1}, x\right)$ & $x^{6}+6 x^{4}+9 x^{2}$ \\
$\pi\left(G_{1}-u_{1}-v_{1}, x\right)$ & $x^{5}+5 x^{3}+6 x$ \\
\hline \hline
\end{tabular}

TABLE II: THE PERMANENTAL POLYNOMIALS OF $G_{2}$ AND ITS SUBGRAPHS

\begin{tabular}{ll}
\hline$\pi\left(G_{2}, x\right)$ & $x^{8}+9 x^{6}+26 x^{4}+25 x^{2}-4 x+4$ \\
$\pi\left(G_{2}-e_{2}, x\right)$ & $x^{8}+8 x^{6}+20 x^{4}+15 x^{2}$ \\
$\pi\left(G_{2}-w_{1}, x\right)$ & $x^{7}+7 x^{5}+15 x^{3}+10 x$ \\
$\pi\left(G_{2}-z_{1}, x\right)$ & $x^{7}+7 x^{5}+14 x^{3}+6 x$ \\
$\pi\left(G_{2}-w_{1}-z_{1}, x\right)$ & $x^{6}+6 x^{4}+10 x^{2}+4$ \\
\hline \hline
\end{tabular}

\section{The Permanental Polynomials of DegreE SUBDIVISION GRAPHS}

There are various subdivision graphs. In this section we consider the degree subdivision graph. Let $v$ be a vertex of degree $r$ in a graph $H$ and $\left(v, u_{1}\right),\left(v, u_{2}\right), \ldots,\left(v, u_{r}\right)$ the $r$ edges incident with $v$. The graph obtained by inserting $k$ intermediate vertices between the end-vertices $v$ and $u_{i}$ of each edge $\left(v, u_{i}\right)$ is said to be a $k$-degree subdivision graph of $H$ with respect to $v$. Fig. 3 illustrates the 1-degree subdivision graph. The permanental polynomial of the 1-degree subdivision graph will be deduced as follows. 

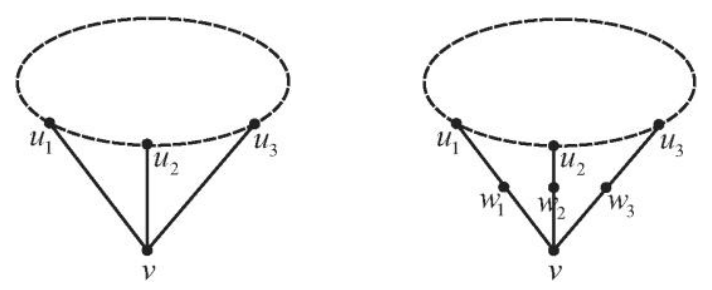

Fig. 3. The graph and its 1 -degree subdivision graph with respect to vertex $v$.

Theorem III.1 Let $v$ be a vertex of degree three in $H$ and $u_{1}, u_{2}$ and $u_{3}$ the three neighbors of $v$. The graph $G$ is the 1 -degree subdivision graph of $H$ by inserting the vertex $w_{i}$ between the end-vertices $v$ and $u_{i}$ of each edge $\left(v, u_{i}\right)$. Let

$\beta\left(H, v, u_{1}, u_{2}, u_{3}\right)$ be the column vector $(\pi(H, x), \pi(H-v$,

$x), \pi\left(H-v-u_{1}, x\right), \pi\left(H-v-u_{2}, x\right), \pi\left(H-v-u_{3}, x\right), \pi(H$ $\left.-v-u_{2}-u_{3}, x\right), \pi\left(H-v-u_{1}-u_{3}, x\right), \pi\left(H-v-u_{1}-u_{2}, x\right)$, $\pi\left(H-v-u_{1}-u_{2}-u_{3}, x\right)$. Then

$$
\beta\left(G, v, w_{1}, w_{2}, w_{3}\right)=A_{3} \cdot \beta\left(H, v, u_{1}, u_{2}, u_{3}\right),
$$

where $A_{3}$ is a $9 \times 9$ matrix whose $i$-th row vector is $s_{i}$ for $1 \leq i \leq 9$. Explicitly, $s_{1}=\left(1, x^{4}+3 x^{2}-x, x^{3}+2 x-1, x^{3}\right.$ $\left.+2 x-1, x^{3}+2 x-1, x^{2}+1, x^{2}+1, x^{2}+1,1\right) ; s_{2}=\left(0, x^{3}, x^{2}\right.$, $\left.x^{2}, x^{2}, x^{2}, x, x, x, 1\right) ; s_{3}=\left(0, x^{2}, 0, x, x, 1,0,0,0\right) ; s_{4}=\left(0, x^{2}\right.$, $x, 0, x, 0,1,0,0) ; s_{5}=\left(0, x^{2}, x, x, 0,0,0,1,0\right) ; s_{6}=(0, x, 1,0,0,0$, $0,0,0) ; s_{7}=(0, x, 0,1,0,0,0,0,0) ; s_{8}=(0, x, 0,0,1,0,0,0,0)$; $s_{9}=(0,1,0,0,0,0,0,0,0)$.

Proof: By Theorem I.2, it follows that

$$
\begin{gathered}
\pi(G, x)=x \pi(G-v, x)+\pi\left(G-v-w_{1}, x\right) \\
+\pi\left(G-v-w_{2}, x\right)+\pi\left(G-v-w_{3}, x\right) \\
+2 \sum_{C \in \Gamma_{v}(G)}(-1)^{|V(C)|} \pi(G-V(C), x) .
\end{gathered}
$$

In the same approach, we can obtain that

$$
\begin{gathered}
\pi(G-v, x) \\
=x \pi\left(G-v-w_{1}, x\right)+\pi\left(G-v-w_{1}-u_{1}, x\right) \\
=x\left[x \pi\left(G-v-w_{1}-w_{2}, x\right)\right. \\
\left.+\pi\left(G-v-w_{1}-w_{2}-u_{2}, x\right)\right]+\pi\left(G-v-w_{1}-u_{1}, x\right) \\
=x^{2}\left[x \pi\left(G-v-w_{1}-w_{2}-w_{3}, x\right)\right. \\
\left.+\pi\left(G-v-w_{1}-w_{2}-w_{3}-u_{3}, x\right)\right] \\
+x \pi\left(G-v-w_{1}-w_{2}-u_{2}, x\right)+\pi\left(G-v-w_{1}-u_{1}, x\right) \\
=x^{3} \pi(H-v, x)+x^{2} \pi\left(H-v-u_{3}, x\right) \\
+x \pi\left(G-v-w_{1}-w_{2}-u_{2}, x\right)+\pi\left(G-v-w_{1}-u_{1}, x\right) .
\end{gathered}
$$

Moreover,

$$
\pi\left(G-v-w_{1}-w_{2}-u_{2}, x\right)
$$

$$
\begin{gathered}
=x \pi\left(G-v-w_{1}-w_{2}-u_{2}-w_{3}, x\right) \\
+\pi\left(G-v-w_{1}-w_{2}-u_{2}-w_{3}-u_{3}, x\right) \\
=x \pi\left(H-v-u_{2}, x\right)+\pi\left(H-v-u_{2}-u_{3}, x\right)
\end{gathered}
$$

and

$$
\begin{gathered}
\pi\left(G-v-w_{1}-u_{1}, x\right) \\
=x \pi\left(G-v-w_{1}-u_{1}-w_{2}, x\right) \\
+\pi\left(G-v-w_{1}-u_{1}-w_{2}-u_{2}, x\right) \\
=x\left[x \pi\left(G-v-w_{1}-u_{1}-w_{2}-w_{3}, x\right)\right. \\
\left.+\pi\left(G-v-w_{1}-u_{1}-w_{2}-w_{3}-u_{3}, x\right)\right] \\
+\left[x \pi\left(G-v-w_{1}-u_{1}-w_{2}-u_{2}-w_{3}, x\right)\right. \\
\left.+\pi\left(G-v-w_{1}-u_{1}-w_{2}-u_{2}-w_{3}-u_{3}, x\right)\right] \\
=x^{2} \pi\left(H-v-u_{1}, x\right)+x\left[\pi\left(H-v-u_{1}-u_{3}, x\right)\right. \\
\left.+\pi\left(H-v-u_{1}-u_{2}, x\right)\right]+\pi\left(H-v-u_{1}-u_{2}-u_{3}, x\right) .
\end{gathered}
$$

Combining (5), (6), and (7), we derive that

$$
\pi(G-v, x)=s_{2} \cdot \beta\left(H, v, u_{1}, u_{2}, u_{3}\right) .
$$

Based on the result of Theorem I.2, we can get that

$$
\begin{gathered}
\pi\left(G-v-w_{1}, x\right) \\
=x^{2} \pi(H-v, x)+x \pi\left(H-v-u_{2}, x\right) \\
+x \pi\left(H-v-u_{3}, x\right)+\pi\left(H-v-u_{2}-u_{3}, x\right) ; \\
\pi\left(G-v-w_{2}, x\right) \\
+x \pi\left(H-v-u_{3}, x\right)+\pi\left(H-v-u_{1}-u_{3}, x\right) \\
\pi\left(G-v-w_{3}, x\right) \\
\pi(H-v, x)+x \pi\left(H-v-u_{1}, x\right) \\
=x \pi\left(H-v-u_{2}, x\right)+\pi\left(H-v-u_{1}-u_{2}, x\right) .
\end{gathered}
$$

Similarly,

$$
\begin{gathered}
\quad \pi\left(G-v-w_{2}-w_{3}, x\right) \\
=x \pi(H-v, x)+\pi\left(H-v-u_{1}, x\right) ; \\
\pi\left(G-v-w_{1}-w_{3}, x\right) \\
=x \pi(H-v, x)+\pi\left(H-v-u_{2}, x\right) ; \\
\quad \pi\left(G-v-w_{1}-w_{2}, x\right) \\
=x \pi(H-v, x)+\pi\left(H-v-u_{3}, x\right) .
\end{gathered}
$$

Since

$$
\begin{gathered}
\pi(H, x)-x \pi(H-v, x)-\pi\left(H-v-u_{1}, x\right) \\
-\pi\left(H-v-u_{2}, x\right)-\pi\left(H-v-u_{3}, x\right) \\
=2 \sum_{C \in \Gamma_{v}(H)}(-1)^{|V(C)|} \pi(H-V(C), x)
\end{gathered}
$$

and 


$$
\begin{aligned}
& \sum_{C \in \Gamma_{v}(H)}(-1)^{|V(C)|} \pi(H-V(C), x) \\
= & \sum_{C \in \Gamma_{v}(G)}(-1)^{|V(C)|} \pi(G-V(C), x) .
\end{aligned}
$$

Substituting these two equalities into (4) and then combining (4), (8) - (11), we can deduce that

$$
\pi(G, x)=s_{1} \cdot \beta\left(H, v, u_{1}, u_{2}, u_{3}\right)
$$

$$
\begin{array}{llr}
\text { It } \quad \text { is } \quad \text { easy } \quad \text { to } \quad \text { see } & \text { that } \\
\pi\left(G-v-w_{1}-w_{2}-w_{3}, x\right)=\pi(H, x) . & & \text { Thus } \\
\beta\left(G, v, w_{1}, w_{2}, w_{3}\right)=A_{3} \cdot \beta\left(H, v, u_{1}, u_{2}, u_{3}\right) \text { holds. } &
\end{array}
$$

Remark III.2 For a graph $H$ with a vertex $v$ of degree three, if $G$ is a $k$-degree subdivision graph of $H$ with respect to $v$, then the permanental polynomial of $G$ can be obtained from the permanental polynomials of $H$ and its subgraphs recursively, i.e.

$$
\beta\left(G, v, w_{1}, w_{2}, w_{3}\right)=A_{3}{ }^{k} \cdot \beta\left(H, v, u_{1}, u_{2}, u_{3}\right) .
$$

The result of Theorem III.1 can be generalized to the case that $v$ is of degree $r(r \geq 3)$, but in this case the order of the iterative matrix is larger.

\section{CONCLUSION}

This paper provides methods to compute the permanental polynomials of subdivision graphs in a recursive way. These methods come from linear algebra, and they are efficient and convenient. Except subdivision graphs, such methods can also be used to derive the permanental polynomials of graphs under other graph operations, such as gluing and splicing operations.

\section{REFERENCES}

[1] M. Loebl and I. Moffatt, "A permanent formula for the jones polynomial," Adv. Appl. Math., vol. 47, pp. 659 - 667, October 2011.

[2] T. Rudolph, "A simple encoding of a quantum circuit amplitude as a matrix permanent," Phys. Rev. A, vol. 80, 054302, November 2009.

[3] G. G. Cash, "The permanental polynomial," J. Chem. Inf. Comput. Sci., vol. 40, pp. 1203-1206, July 2000.

[4] G. G. Cash, "Permanental polynomials of smaller fullerenes," J. Chem. Inf. Comput. Sci., vol. 40, pp. 1207-1209, July 2000.

[5] L. G. Valiant, "The complexity of computing the permanent," Theor. Comput. Sci., vol. 8, pp. 189-201, 1979.

[6] H. Minc, Permanents, 1st ed., London: Addision-Wesley, 1978, ch. 1, pp. 1.

[7] R. M. Cvetković, M. Doob, and H. Sachs, Spectra of graphs, 1st ed., New York: Academic Press, 1980, ch. 1, pp. 32-34.

[8] R. Merris, K. R. Rebman, and W. Watkins, "Permanental polynomials of graphs," Linear Algebra. Appl., vol. 38, pp. 273-288, June 1981.

[9] M. Borowiecki and T. Jóźwiak, "Computing the permanental polynomial of a multigraph,” Discuss. Math., vol. 5, pp. 9-16, 1982.

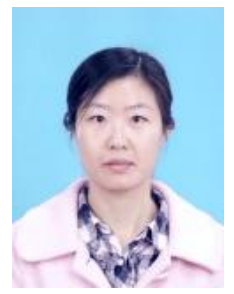

Wei Li was born in Liaoning Province, China in March 1984. Li was awarded the doctor of science degree on applied mathematics and bachelor of science degree on information and computational science in 2012 and 2007 from Lanzhou University in Lanzhou, China.

She has been working for the Department of Applied Mathematics at Northwestern Polytechnical University in a lecturer position from 2012 to now. She has published five papers on graph theory, such as computing the permanental polynomials of bipartite graphs by Pfaffian orientation (USA Philadelphia: Elsevier, 2012), dimers statistics of honeycomb lattices on klein bottle, mobius strip and cylinder (USA Philadelphia: Elsevier, 2012). Her research focuses on the permanental polynomials of graphs and matching problems 